\title{
Knockdown of B7-H6 inhibits tumor progression and enhances chemosensitivity in B-cell non-Hodgkin lymphoma
}

\author{
FEIFEI WU, JING WANG and XIAOYAN KE
}

Department of Hematology and Lymphoma Research Center, Peking University Third Hospital, Beijing 100191, P.R. China

Received December 15, 2015; Accepted January 20, 2016

DOI: $10.3892 /$ ijo.2016.3393

\begin{abstract}
B7 homologue 6 (B7-H6) is a new member of the B7 family molecules and is selectively expressed on tumor cells, especially in hematologic malignancies. However, the role of B7-H6 in lymphoma progression and chemosensitivity remains unclear. We determined the effects of downregulating B7-H6 expression on tumorigenesis and chemosensitivity in B-cell lymphoma. Stable B7-H6 knockdown in CA46 cells was established with a lentiviral system. The expression of mRNA was measured by PCR while protein expression was detected by western blotting and flow cytometry. Cell viability, apoptosis and cell cycle distribution were analyzed using CCK-8, colony formation and flow cytometry assays, respectively. Cell migration and invasion were determined using the Transwell chamber assay. B7-H6 was widely expressed in B-cell lymphomas. Knockdown of B7-H6 inhibited cell proliferation, colony formation and migration and invasion of lymphoma cells. After B7-H6 silencing, CA46 cells were arrested in G0/G1 phase. Moreover, the silencing of B7-H6 increased cell apoptosis and sensitivity to vincristine and dexamethasone. Investigation of expression of downstream targets of STAT3 supported a theory in which B7-H6 knockdown may confer an antitumor effect via abrogation of the STAT3 pathway. This study demonstrates that B7-H6 plays an important role in the pathogenesis and chemosensitivity of lymphoma. B7-H6 is therefore a potential clinical biomarker and therapeutic target in B-cell lymphomas.
\end{abstract}

Correspondence to: Professor Xiaoyan Ke, Department of Hematology and Lymphoma Research Center, Peking University Third Hospital, 49 North Garden Road, Haidian, Beijing 100191, P.R. China E-mail: bysydrke@126.com

Abbreviations: B7-H6, homologue 6; NHL, non-Hodgkin lymphoma; FBS, fetal bovine serum; VCR, vincristine; Dex, dexamethasone; PCNA, proliferating cell nuclear antigen

Key words: B7 homologue 6, non-Hodgkin lymphoma, cancer therapy, chemosensitivity

\section{Introduction}

Non-Hodgkin lymphoma (NHL) is the most common hematologic malignancy arising from lymphoid tissue worldwide (1), $\sim 85 \%$ of which derive from B lymphocytes. The overall survival of B-cell lymphomas has significantly improved since the FDA approval and clinical use of rituximab, a monoclonal antibody targeting CD20-positive malignant B cells (2). However, relapse and resistance are obstacles to the cure of B-NHL patients, underscoring the need for more effective therapies. Immunotherapy is one promising new direction for lymphoma as it is for many other malignancies (2).

The B7 family is a group of molecules important for the regulation of tumor immunity, and can profoundly affect tumor progression and elimination (3-8). Monoclonal antibodies targeting B7 molecules including CD80 (9), PD-L1 (10), PD-L2 (11) and B7-H3 (12), have shown promising results in lymphomas, melanoma, non-small cell lung cancer and other cancers. B7-H6, also called NCR3L1 or DKFZp686024166, is a new member of the $\mathrm{B} 7$ family identified by Brandt et al in 2009 (3). B7-H6 is a 454-aa-long type I transmembrane protein that shows considerable homology with PD-L1 and B7-H3 (3), both of which contribute to the oncogenesis and chemoresistance of lymphomas $(13,14)$. The receptor of B7-H6, NKp30, is an activating NK-cell receptor. The interaction of tumor membranebound B7-H6 and NKp30 leads to the activation of NK cells and to the lysis of tumor cells $(3,15)$. A fusion protein of B7-H6 ectodomain and CD20 single-chain fragment stimulates NKp30mediated NK cell cytotoxicity (7). Moreover, the NKp30-based chimeric antigen receptor promotes T-cell effector functions and antitumor efficacy through recognizing and killing B7-H6positive tumor cells (16). These results indicate that B7-H6 may be a potential therapeutic target in cancer therapy.

It is reported that B7-H6 is widely and specifically expressed on tumor cells including melanomas $(3,17)$, cervical carcinomas (3), ovarian carcinomas $(3,4)$, liver cancer (18), gastric cancer (19), lung cancer (20), neuroblastoma (6), T and B lymphomas and myeloid leukemia $(3,8)$, but is absent from normal tissues (3). B7-H6 is associated with tumor progression and metastasis in ovarian cancers (4). To date, however, little is known about the oncogenic role of B7-H6 in lymphomas. Hence, we established a B7-H6 knockdown cell line for the first 
time to investigate the impact of B7-H6 on cell proliferation, apoptosis, cell cycle, migration, invasion and chemosensitivity. The underlying mechanisms were also investigated, with the aim of providing a basis for clinical therapy.

\section{Materials and methods}

Cell lines and cell culture. Raji, CA46, Z138, Maver and HeLa cell lines were obtained from the American Type Culture Collection (ATCC, Rockville, MD, USA). Raji, CA46 and Z138 cells were cultured in RPMI-1640 medium (Gibco, Grand Island, NY, USA) supplemented with $2 \mathrm{mM}$ L-glutamine, 10\% FBS (Hyclone, South Logan, UT, USA), $100 \mathrm{U} / \mathrm{ml}$ penicillin and $100 \mu \mathrm{g} / \mathrm{ml}$ streptomycin. Maver and HeLa cells were respectively cultured in IMDM and DMEM medium. Cells were routinely cultured at $37^{\circ} \mathrm{C}$ in a humidified incubator containing $5 \% \mathrm{CO}_{2}$ (14). The primary tumor proteins were obtained from Peking University Third Hospital. The study was approved by the local ethics committee and all patients provided written informed consent.

Lentivirus-based RNA interference transfection and generation of stable cell lines. The human B7-H6 targeting small hairpin RNA (shRNA) was designed and synthesized by Life Technologies (Lifetech, Beijing, China). The sequence was 5'-CGGCACAGTCTTTCTGAAACT-3'. A negative non-target control shRNA was also used with the sequence 5'-CAAC AAGATGAAGAGCACCAA-3'. These shRNAs were used to generate recombinant lentiviral particles as described (14). CA46 cells were infected in the presence of $8 \mu \mathrm{g} / \mathrm{ml}$ of polybrene (Sigma, Milwaukee, WI, USA) and selected by flow cytometry $72 \mathrm{~h}$ later. The B7-H6 knockdown was confirmed by RT-PCR, western blotting and flow cytometry. The infected cells comprised B7-H6shRNA (CA46shB7-H6) and nontargeted control (CA46shCtrl) groups, while non-infected cells (CA46) constituted an additional control group. These three cell lines were used for the following experiments.

RT-PCR and quantitative PCR. Total RNA was extracted using TRIzol reagent (Invitrogen, Carlsbad, CA, USA) and reverse transcribed to cDNA using Reverse Transcription System (Promega, Madison, WI, USA) according to the manufacturer's instructions. cDNA (100 ng) was subjected to PCR amplification in a total volume of $25 \mu \mathrm{l}$ according to the manufacturer's protocols. The primer sequences of the PCR were as follows: B7-H6 forward, 5'-ACAGTAAATGCCTGATGGACCT-3'; B7-H6 reverse, 5'-ATTGGGTATGTGAATGCTGGT-3'; $\beta$-actin forward, 5'-TGACGTGGACATCCGCAAAG-3'; $\beta$-actin reverse, 5'-CTGGAAGGTGGACAGCGAGG-3'. The PCR products were run on agarose gels and visualized under ultraviolet. Quantitative PCR was conducted with SuperReal PreMix Plus kit (Tiangen, Beijing, China) in a volume of $20 \mu 1$ according to the manufacturer's instructions. The primer sequences of quantitative PCR were as follows: B7-H6 forward, 5'-GCACTTCCTCACCGCTAATG-3'; B7-H6 reverse, 5'-AGCCTGTTTCCTTTCGCTATT-3'. The ratio of expression of B7-H6 to $\beta$-actin mRNA was calculated.

Western blotting. Cytoplasmic proteins were extracted from primary and culture cells by lysis buffer composed of $50 \mathrm{mmol} / \mathrm{l}$
Tris- $\mathrm{HCl}$ (pH 7.5), $150 \mathrm{mmol} / \mathrm{l} \mathrm{NaCl}, 5 \mathrm{mmol} / \mathrm{l}$ EDTA, $0.5 \%$ Nonidet P-40, $5 \mathrm{mmol} / \mathrm{l}$ dithiothreitol and $10 \mathrm{mmol} / \mathrm{l} \mathrm{NaF}$, and containing protease inhibitor cocktail (Applygen, Beijing, China). Proteins were separated by SDS-PAGE and transferred onto nitrocellulose membranes. After blocking, the membranes were sequentially incubated with primary antibodies, including anti-B7-H6, anti-c-Myc, anti-Rb, and anti-phospho-Rb (Abcam, Cambridge, MA, USA); anti-Survivin, anti-CDK4, anti-CDK6, anti-p21, anti-Bad, anti-MMP-2, anti-MMP-9, and anti- $\beta$-actin (Santa Cruz Biotechnology, Santa Cruz, CA, USA); and antiCyclin D1, anti-Bcl-2, anti-Bcl-xL, anti-Bax, anti-Caspase-3, anti-Caspase-8, anti-STAT3, anti-phospho-STAT3 (Ser727 and Tyr705), anti-ERK1/2 and phospho-ERK1/2 (CST, Beverly, MA, USA), and then with anti-rabbit or mouse secondary antibodies (LI-COR, Lincoln, NE, USA). Fluorescent bands were visualized using an Odyssey infrared imaging system (LI-COR).

Flow cytometry. Single cell suspensions were stained with B7-H6-APC or IgG1-APC (R\&D, Minneapolis, MN, USA) antibodies on ice for $30 \mathrm{~min}$, cells were analyzed using a flow cytometry system (FACSCalibur; BD Biosciences, San Jose, CA, USA).

Colony formation assay. An 800 single-cell suspension was resuspended in $1 \mathrm{ml}$ medium with 20\% FBS and 0.9\% methylcellulose medium (Sigma, St. Louis, MO, USA). Samples were plated in 24-well plates and incubated for 14 days. A colony with $>50$ cells was counted as a positive colony. The colonyforming ability was calculated to be the number of colonies in the test group expressed as a percentage of the number of colonies in the control group (13).

CCK-8 assay. The Cell Counting Kit-8 (CCK-8; Dojindo, Tokyo, Japan) was used to study cell viability according to the manufacturer's instructions. A cell suspension was inoculated into a 96-well plate ( $2 \times 10^{4}$ cells/well) and incubated for 0,24 , 48 or $72 \mathrm{~h}$. At every time-point, $10 \mu \mathrm{lCCK}-8$ was added to each well and the plate was incubated for $3 \mathrm{~h}$ at $37^{\circ} \mathrm{C}$ and $5 \% \mathrm{CO}_{2}$. Absorbance was measured at $450 \mathrm{~nm}$ using a microplate reader.

For cell viability assay, cells were seeded in 96-well plates and exposed to various concentrations of $\operatorname{VCR}(0,1.5,3,6$, $12.5,25,50,75$ and $100 \mu \mathrm{g} / \mathrm{ml})$ or Dex $(0,1.5,3,6,12.5,25$, $50,100,200,400,800,1,200,1,600$ and 2,400 $\mu \mathrm{g} / \mathrm{ml}$ ) for $24 \mathrm{~h}$. A CCK-8 assay was used to detect the chemotherapeutic sensitivity of cells. The assay was performed with six replicates $(n=6)$ for each group and repeated at least three times.

Cell apoptosis analysis. To explore the effect of B7-H6 knockdown on cell apoptosis, cells were serum starved for $24 \mathrm{~h}$. To explore the B7-H6 knockdown on the sensitivity to VCR and Dex, cells were seeded into 6-well plates at a cell density of $1 \times 10^{6}$ cells/well and treated with or without VCR $(25 \mu \mathrm{g} / \mathrm{ml})$ or Dex $(200 \mu \mathrm{g} / \mathrm{ml})$ for $24 \mathrm{~h}$. Cells were then harvested and apoptosis was analyzed by FACSCalibur cytometer using Annexin V-APC and 7-AAD (BD Pharmingen, San Jose, CA, USA) according to the manufacturer's instructions. CellQuest software was used for data analysis.

Cell cycle analysis. To explore the effect of B7-H6 knockdown on the cell cycle, cells were serum starved for $24 \mathrm{~h}$. Cells were 
A

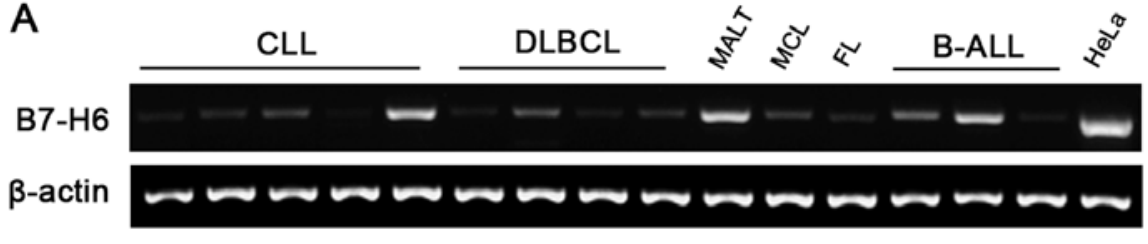

B $\mathrm{B} 7-\mathrm{H} 6$

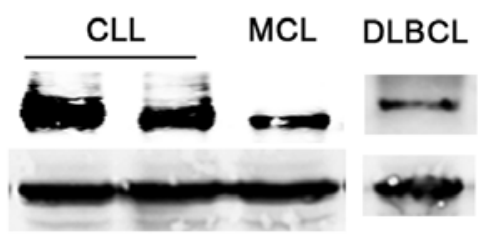

C
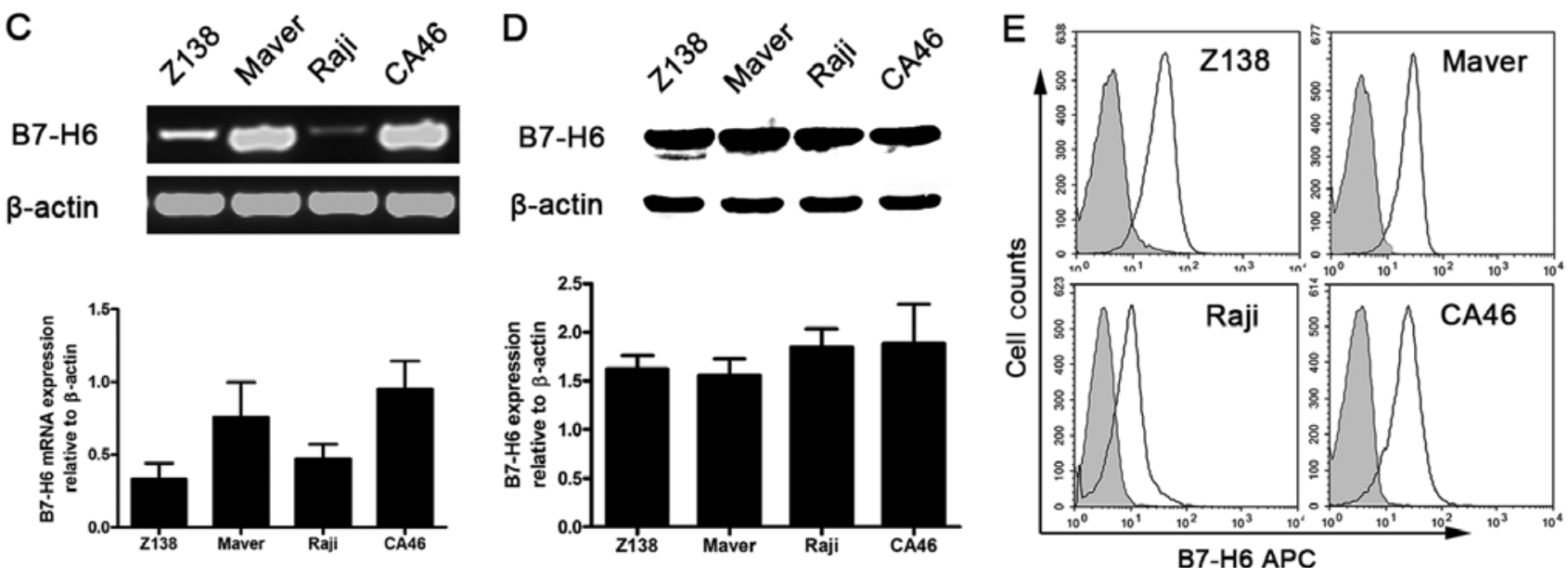

Figure 1. B7-H6 expression in human primary B-cell lymphomas and cell lines. (A) B7-H6 is widely expressed in B-cell lymphomas including CLL, DLBCL, MALT, MCL, FL and B-ALL at mRNA level using HeLa cell line as a positive control. (B) B7-H6 is expressed in CLL, MCL and DLBCL at protein level. (C) B7-H6 is expressed in Raji, CA46, Z138 and Maver cell lines at mRNA level. (D) B7-H6 is expressed at nuclear and cytosolic protein level and (E) membrane level.

then collected, washed and fixed in $70 \%$ ethanol overnight at $4^{\circ} \mathrm{C}$. Cellular DNA was stained with propidium iodide (PI) (Beyotime, Nangtong, China) in the dark for $10 \mathrm{~min}$ at room temperature. DNA content and cell number were determined using a FACSCalibur cytometer. The data were analyzed using the ModFit program (Verify Software House, Cambridge, UK).

Cell migration and invasion assay. Migration and invasion assays were performed using 24-well Transwell cell culture chambers (Corning, NY, USA) or Matrigel-coated invasion chambers. Cells $\left(5 \times 10^{5}\right.$ cells/well $)$ were serum starved for $24 \mathrm{~h}$ and placed in the upper chamber in serum-free medium. Medium containing 10\% FBS was placed in the lower chamber as a chemoattractant. After $24 \mathrm{~h}$ of incubation, the migrated cells in the lower chamber were collected and resuspended. Non-invading cells were removed with a cotton-tipped swab from the top of Matrigel and invading cells were fixed and stained with $0.1 \%$ crystal violet. Cells were photographed and counted in six random microscopic fields.

Statistical analysis. Statistical analysis was performed using SPSS 18.0 software (Chicago, IL, USA). Data are shown as mean \pm SD of triplicate for each experiment. Differences between groups were evaluated using SPSS 18.0 software. Data are shown as mean \pm SD of all replicates for each experiment. Differences between groups were evaluated using ANOVA. $\mathrm{P}<0.05$ was considered to be statistically significant.

\section{Results}

B7-H6 is expressed in B-cell non-Hodgkin lymphomas. Studies have shown that B7-H6 mRNA is absent in normal tissues and relatively abundant in tumor cells (3). To investigate the role of B7-H6 in B-cell NHL, we first detected B7-H6 expression at the mRNA level in patients with B-cell NHL and healthy donors. Using the HeLa cell line as a positive control, we found that B7-H6 was widely and heterogeneously expressed in B-cell NHLs including chronic lymphocytic leukemia (CLL), diffuse large B-cell lymphoma (DLBCL), mucosa-associated lymphoid tissue (MALT) lymphoma, mantle cell lymphoma (MCL), follicular lymphoma (FL) and B-cell acute lymphocytic lymphoma (B-ALL) (Fig. 1A), but was absent in healthy donors' peripheral mononuclear cells (PMNC) at the mRNA level (data not shown). We also measured the expression of B7-H6 at nuclear/cytosolic level in 8 B-cell NHL patients including CLL $(n=3)$, DLBCL $(n=3)$, MCL $(n=1)$ and B-ALL $(n=1)$. B7-H6 expression was observed in tumor cells from 4 patients ( 2 patients with CLL, 1 patient with MCL and 1 patient with DLBCL; Fig. 1B). However, we did not detect B7-H6 in PMNC of 4 healthy donors (data not shown). Furthermore, human B-cell lymphoma cell lines (Raji, CA46, Z138 and Maver) expressed B7-H6 at the level of mRNA (Fig. 1C), nuclear/cytosolic protein (Fig. 1D) and membrane protein (Fig. 1E). These results indicated that B7-H6 is widely expressed in B-cell lymphoma specimen and cell lines.

The establishment of a B7-H6 stable knockdown cell line. To investigate the role of B7-H6 in B-cell lymphoma, CA46 cells were selected for in vitro studies as they expressed a high and steady level of endogenous B7-H6 (Fig. 1C-E). B7-H6 knockdown in CA46 cells was performed using lentivirus transduction to stably express shRNA targeting B7-H6. There was no significant difference of B7-H6 expression between 

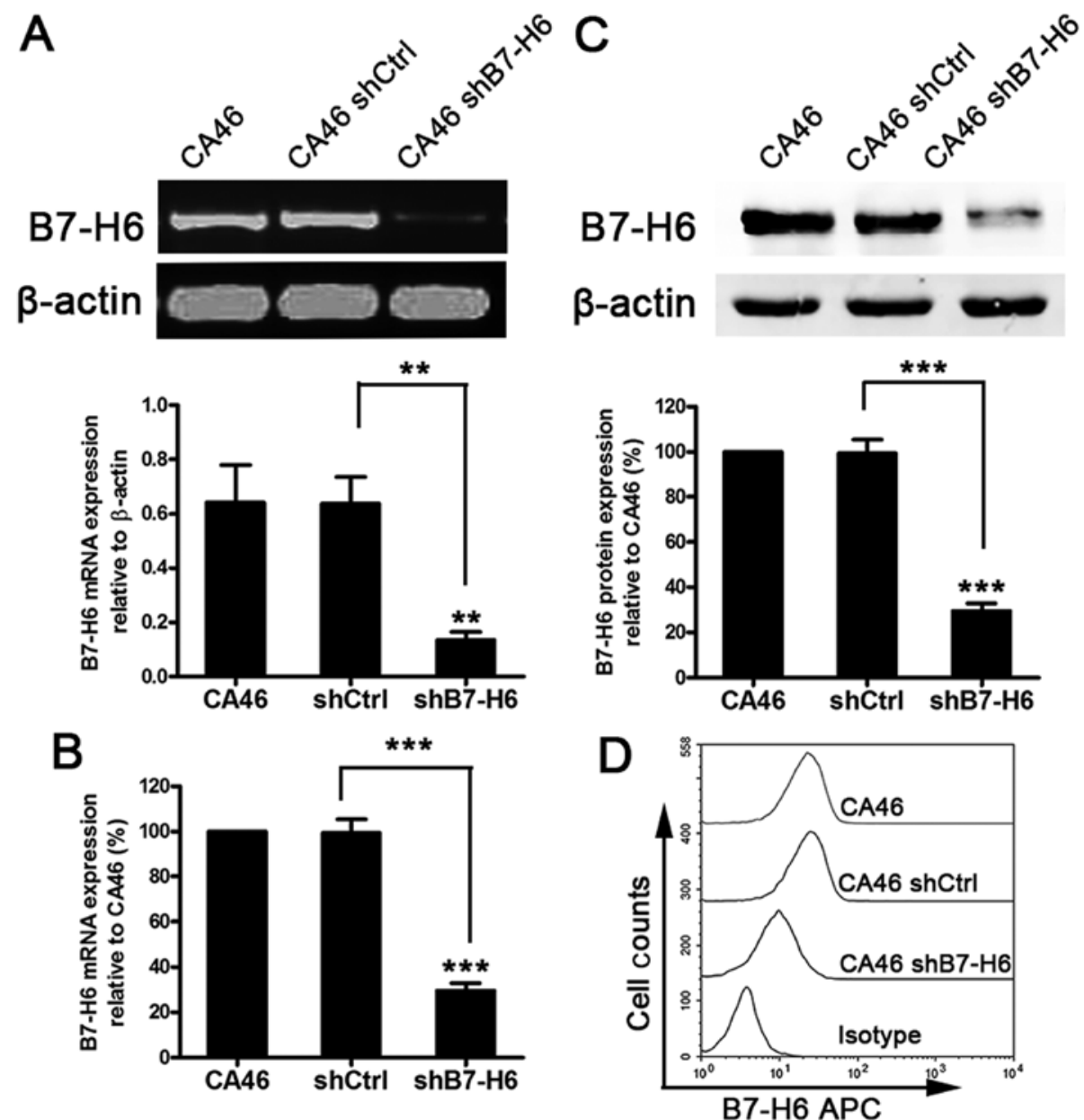

Figure 2. The effect of silencing B7-H6 by shRNA in CA46 cells. (A) B7-H6 mRNA knockdown is shown by RT-PCR and (B) quantitative PCR. (C) B7-H6 nuclear and cytoplasmic protein was knocked down as determined by western blotting. $\beta$-actin was probed as a control. (D) B7-H6 membrane protein knockdown was determined by flow cytometry. The data are from at least three separate experiments. ${ }^{* * *} \mathrm{P}<0.01,{ }^{* * *} \mathrm{P}<0.001$ compared to the control group.

A

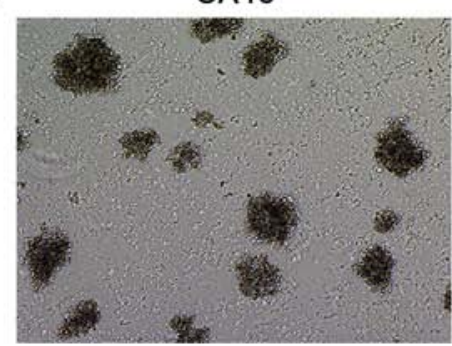

B

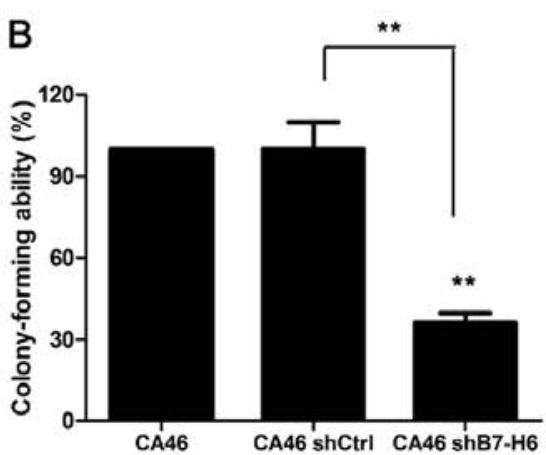

CA46 shCtrl
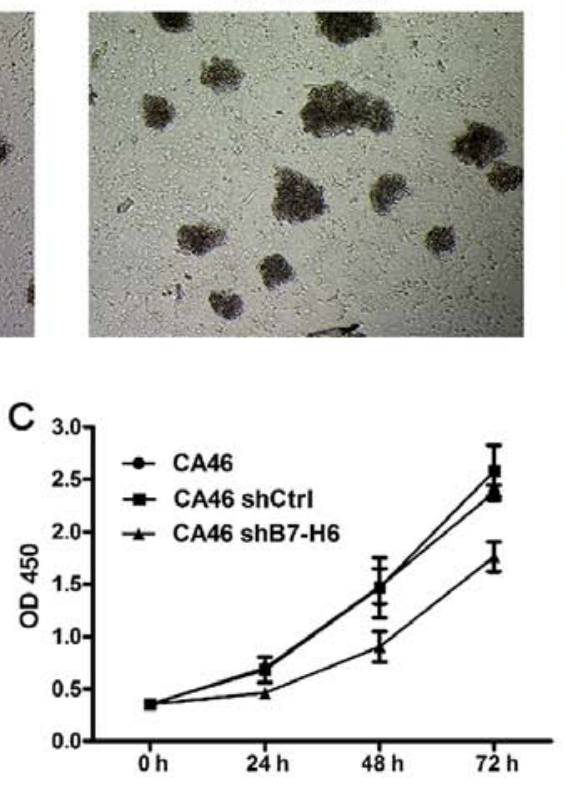

CA46 shB7-H6

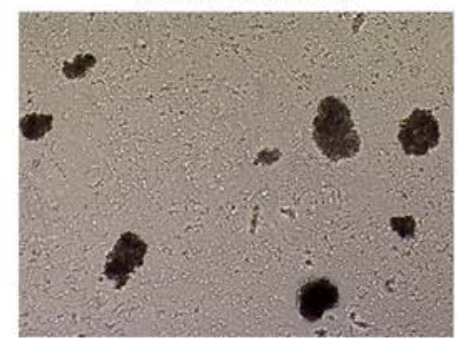

D

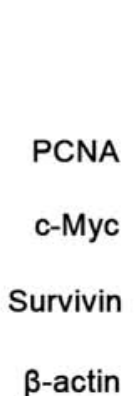

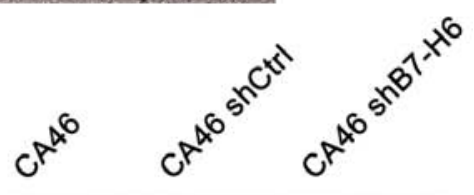
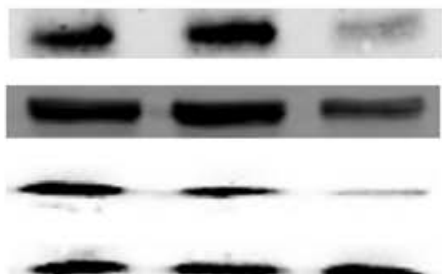

Figure 3. B7-H6 knockdown inhibits tumor proliferation. (A) B7-H6 knockdown inhibited the colony formation detected by methyl cellulose colony forming assay (x100). (B) Quantification of colony was shown with or without knockdown of B7-H6. (C) B7-H6 silencing inhibited the cell growth analyzed by CCK-8 assay. (D) PCNA, c-myc and survivin protein levels were examined by western blotting using $\beta$-actin as a loading control. Data are presented as means \pm SD of at least three independent experiments. ${ }^{* *} \mathrm{P}<0.01$ compared to the control group. 


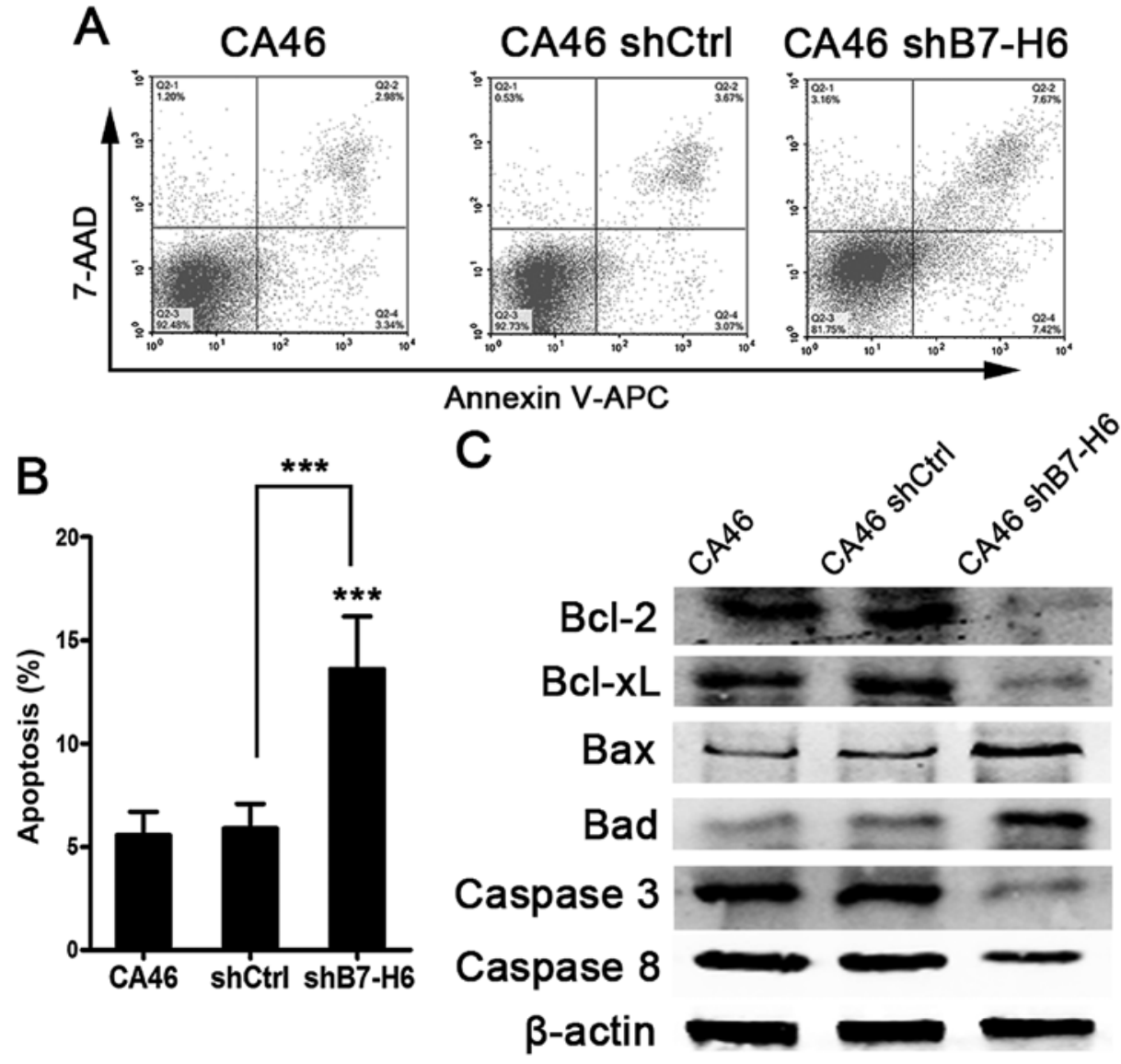

CA46 shCtrl CA46 shB7-H6

Figure 4. B7-H6 is associated with tumor apoptosis. (A) B7-H6 knockdown increased tumor apoptosis by serum starvation. Annexin $\mathrm{V}^{+} / 7-\mathrm{AAD}^{-}$and Annexin $\mathrm{V}^{+} / 7-\mathrm{AAD}^{+}$were designed as early stage and advanced stage of the apoptotic process. (B) Apoptotic rates were presented as the percentage of apoptotic cells. The sum of Annexin V-positive cells in the upper right and lower right quandrants represents the total percentage of apoptotic cells. (C) Apoptosis-related protein levels were determined by western blotting with $\beta$-actin as a loading control. Each experiment was repeated at least three times and data are presented as means $\pm \mathrm{SD} .{ }^{* * *} \mathrm{P}<0.001$ compared to the control group.

the CA46 and CA46shCtrl (non-target control shRNA) groups at the mRNA and protein level $(\mathrm{P}>0.05)$. However, B7-H6 expression in the CA46shB7-H6 group containing the B7-H6targetting shRNA, was significantly decreased compared to the CA46 group $(\mathrm{P}<0.01)$. The inhibition rates of mRNA expression in CA46 cells was $74.4 \%$ (Fig. 2A and C), whereas the nuclear/cytosolic protein was reduced by $77.9 \%$ (Fig. 2B) and the membrane protein was inhibited by $60.1 \%$ (Fig. 2D).

B7-H6 knockdown inhibits tumor cell proliferation. To evaluate the effects of B7-H6 knockdown on tumor cell proliferation, colony forming and CCK-8 assays were performed. The colony forming ability of the CA46shB7-H6 group was decreased by $73.8 \%$ compared to the CA46 group $(\mathrm{P}<0.01$; Fig. 3A and B). The cell proliferation of the CA46shB7-H6 group was decreased by $33.8,38.9$ and $25.8 \%$ after 24,48 and $72 \mathrm{~h}$ respectively, compared to the CA46 group $(\mathrm{P}<0.05$; Fig. 3C). Furthermore, we assessed markers reflecting tumor cell proliferation ability. The expressions of PCNA, c-Myc and Survivin in CA46 cells were significantly decreased by 74.1, 21.6 and 55.2\% compared to the CA46shB7-H6 group $(\mathrm{P}<0.05$; Fig. 3D).

B7-H6 knockdown induces apoptosis and inhibits cell cycle progression. To explore the effects of B7-H6 knockdown on cell apoptosis and cell cycle, we analyzed apoptotic rates and cell cycle distribution by flow cytometry. Our results showed that there were $15.09 \%$ apoptotic cells in CA46shB7-H6 cells compared to only $6.32 \%$ in CA46 cells $(\mathrm{P}<0.001$; Fig. 4A and B). We also detected the proteins involved in apoptosis by western blotting. Expression of anti-apoptotic proteins including Bcl-2, Bcl-xL, Caspase-3 and -8 was decreased, and pro-apoptotic proteins including bax and bad were increased in CA46shB7-H6 cells compared to CA46 and CA46shCtrl cells ( $\mathrm{P}<0.05$; Fig. 4C). The percentage of cells in $\mathrm{G} 0 / \mathrm{G} 1$ phase was $43.72 \%$ in CA46 shB7-H6 cells and $28.56 \%$ in CA46 cells ( $\mathrm{P}<0.001$; Fig. $5 \mathrm{~A}$ and $\mathrm{B})$. To further understand the effect of B7-H6 on the cell cycle progression, we measured expression of cell cycle regulatory proteins. Cyclin D1, CDK4, CDK6 and phospho-Rb expression was significantly decreased, while $\mathrm{Rb}$ and $\mathrm{p} 21$ expression was increased in CA46shB7-H6 cells in comparison to CA46 or CA46shCtrl cells $(\mathrm{P}<0.05$; Fig. 5C). The data indicate that knockdown of B7-H6 in CA46 cells increases apoptosis and induces $\mathrm{G} 0 / \mathrm{G} 1$ cycle arrest.

B7-H6 knockdown inhibits tumor cell migration and invasion. We performed Transwell migration and invasion assays to determine whether B7-H6 acts as a regulator of cell migration and invasion. The cell migration assay showed that the 


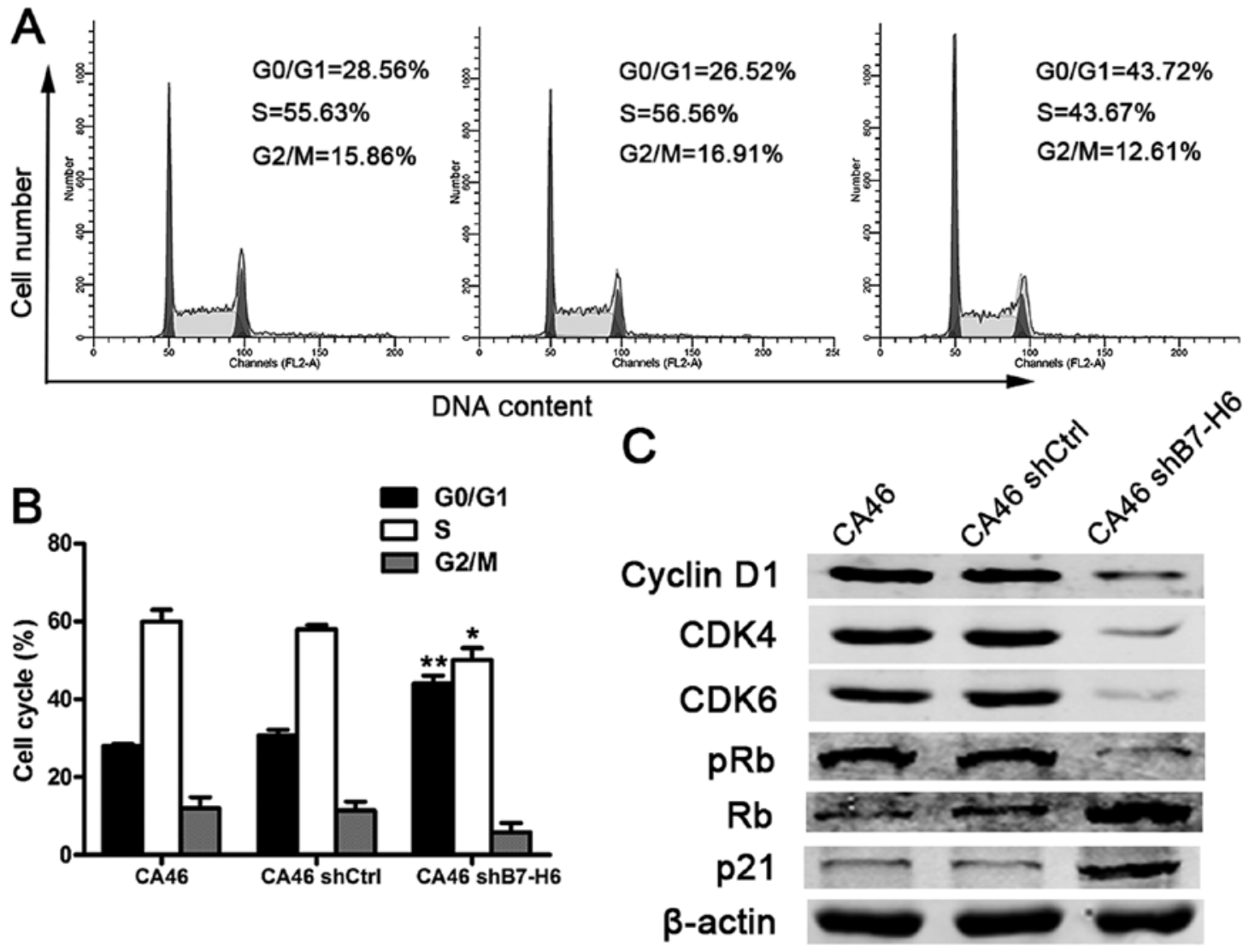

Figure 5. B7-H6 knockdown inhibits cell cycle progression. (A) B7-H6 arrested tumor cell cycle at the G0/G1 phase. (B) The percentage of the cell population at different cell cycle phases is shown. (C) The expression of cell cycle regulatory proteins was measured by western blotting with $\beta$-actin as a loading control Data are presented as means $\pm \mathrm{SD}$ of at least three independent experiments. ${ }^{*} \mathrm{P}<0.05,{ }^{* *} \mathrm{P}<0.01$ compared to the control group.

A

CA46

CA46 shCtrl
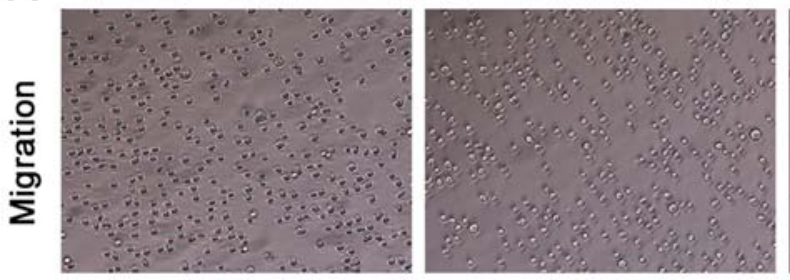

CA46 shB7-H6

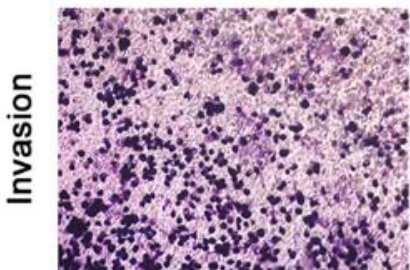

B

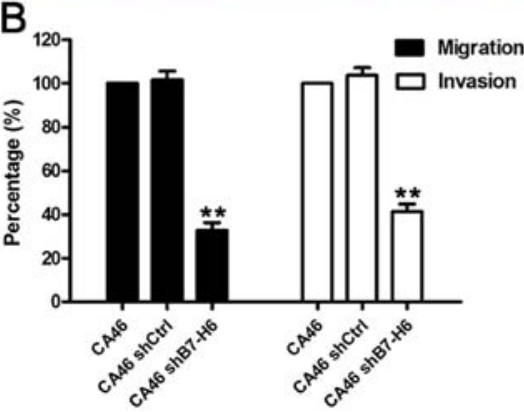

C

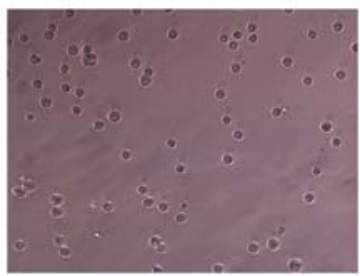

MMP-2

MMP-9

$\beta$-actin

Figure 6. B7-H6 knockdown inhibits tumor migration and invasion. (A) B7-H6 knockdown inhibited tumor migration and invasion capacity (x200). (B) The results are shown as an average of the number of migration and invasion cells from six random microscopic fields. (C) Expression of invasion-related proteins MMP-2 and MMP-9 was decreased in B7-H6 silenced cells, measured by western blotting. Each data point represents means \pm SD of three independent experiments. ${ }^{* *} \mathrm{P}<0.01$ compared to the control group. 

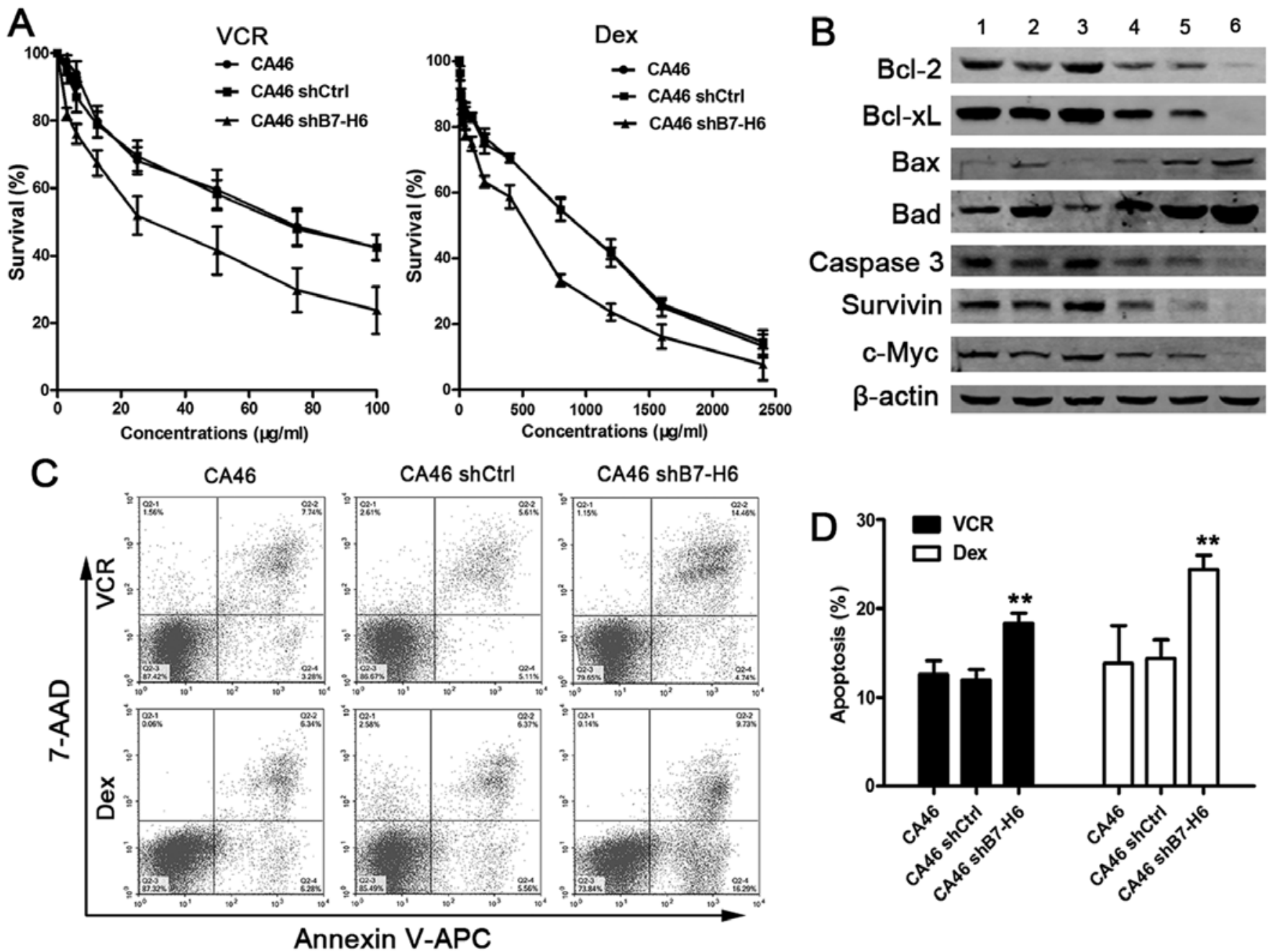

Figure 7. B7-H6 knockdown sensitizes chemotherapy in CA46 cells. (A) Drug concentration-dependent cell survival curves for VCR and Dex for $24 \mathrm{~h}$ in CA46, CA46shCtrl and CA46shB7-H6 cells. (B) Expression of apoptosis-related proteins with or without drugs was detected by western blotting. Lane 1, CA46 control; lane 2, CA46+VCR+Dex; lane 3, CA46shCtrl; lane 4, CA46+VCR+Dex; lane 5, CA46shB7-H6; lane 6, CA46+VCR+Dex). (C) Tumor cells were treated with VCR $(25 \mu \mathrm{g} / \mathrm{ml})$ or Dex $(200 \mu \mathrm{g} / \mathrm{ml})$ for $24 \mathrm{~h}$. Cell apoptosis was significantly increased in CA46shB7-H6 group compared to control group. (D) The results are presented as percentage of apoptotic cells. Each data point represents means \pm SD of three independent experiments. ${ }^{* *} \mathrm{P}<0.01$ compared to the control group.

migration ability was reduced by $67.3 \%$ in CA46shB7-H6 cells compared to CA46 cells $(\mathrm{P}<0.01)$. Furthermore, the invasion assay showed that knockdown of B7-H6 reduced cell invasion ability by $59.4 \%(\mathrm{P}<0.01$; Fig. $6 \mathrm{~A}$ and $\mathrm{B})$. We also measured the expression of crucial proteins involved in cell migration and invasion, and found that expression of MMP-2 and MMP-9 was lower in CA46shB7-H6 cells than in CA46 cells (Fig. 6C). These results indicate that knockdown of B7-H6 could impair cell migration and invasion ability via downregulating the expression of MMP-2 and MMP-9.

B7-H6 knockdown enhances the sensitivity of tumor cells to chemotherapy. To explore whether knockdown of B7-H6 would sensitize lymphoma cells to chemotherapeutic drugs, we chose the frontline drugs in the clinical regimen for NHL, namely VCR and Dex. Cell viability was measured after cells were treated with different concentrations of VCR and Dex for $24 \mathrm{~h}$. The cell survival rates in the CA46shB7-H6 group were significantly decreased compared with CA46 or CA46shCtrl group ( $\mathrm{P}<0.01$; Fig. 7A). We also detected apoptosis induced by VCR and Dex. The data showed that the proportion of apoptotic cells was significantly increased in CA46shB7-H6 cells compared to CA46 cells (19.2 vs. $11.0 \%, \mathrm{P}<0.01$ and 26.0 vs. $12.6 \%, \mathrm{P}<0.01$; Fig. $7 \mathrm{C}$ and $\mathrm{D}$ ). Our previous studies showed that knockdown of B7-H6 inhibited induced cell apoptosis and influenced expression of apoptosis-associated proteins. To verify whether the sensitization of cells to chemotherapeutic drugs was also associated with these proteins, we analyzed the expressions of representative proteins by western blotting. We observed that the expression of Bcl-2, Bcl-xL, Caspase-3, Survivin, and c-Myc was decreased more in drug-treated CA46shB7-H6 group compared to drug-treated CA46 or CA46shCtrl groups (lane 6 vs. lane 2 and lane 6 vs. lane 4; Fig. 7B). Our findings support the notion that sensitization to chemotherapeutic drugs is mediated by apoptosis-related proteins. These results suggest that knockdown of B7-H6 improves the chemosensitivity of B-cell lymphoma cells. 


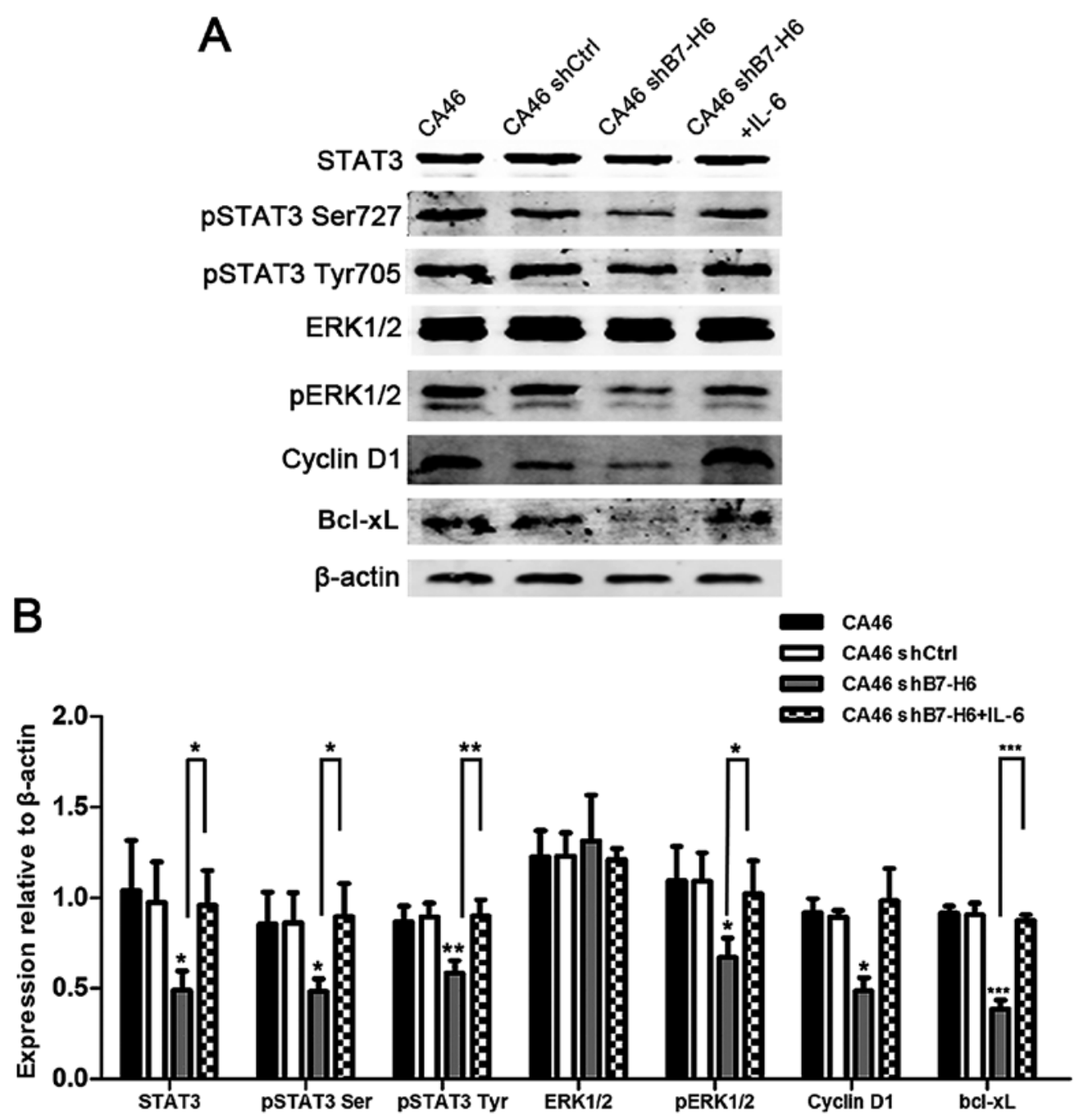

Figure 8. Effects of B7-H6 knockdown on the expression of STAT3 and STAT3 downstream targets. (A) Cellular proteins were analyzed by western blotting in CA46, CA46shCtrl, CA46shB7-H6 and CA46shB7-H6+IL-6 groups with antibodies specific for STAT3, pSTAT3 Ser727, pSTAT3 Tyr705, ERK1/2, pERK1/2, Cyclin D1, Bcl-xL and $\beta$-actin. (B) The ratio of colorimetric density of STAT3 and downstream genes. $\beta$-actin served as loading control. "P $<0.05$, ${ }^{* * *} \mathrm{P}<0.01,{ }^{* * * *} \mathrm{P}<0.001$ compared to the control group.

Knockdown of B7-H6 leads to inactivation of STAT3. STAT3 is often constitutively activated in lymphoma cells (21-23), and is involved in oncogenesis. To evaluate whether loss of STAT3 activation was involved in the effects induced by B7-H6 knockdown, we reactivated STAT3 with IL-6, an activator of STAT3 pathway (24), and detected the expression of its target genes. We observed that expression of pSTAT3, pERK1/2, Cyclin D1 and Bcl-xL was decreased in CA46shB7-H6 cells, but increased by IL- 6 ( $\mathrm{P}<0.05$; Fig. 8$)$, thus indicating that knockdown of B7-H6 leads to inactivation of STAT3.

\section{Discussion}

Immunotherapy has shown some glimmers of hope for the treatment of lymphomas (2). Monoclonal antibodies targeting B7 family checkpoint regulators including PD-L1, B7-H3 and B7-H4 have beneficial effects in reducing tumor burden in melanoma, lung cancer and renal cancer $(10,11)$. B7-H6 is a new member of B7 family and is highly expressed in various tumors $(3,5,18)$ while being absent from normal tissue (3).
Such differential expression makes B7-H6 an attractive therapeutic target. B7-H6 expression is negatively associated with overall survival in ovarian cancer (4) and of modest value as a prognostic marker in non-small cell lung cancer and gastric cancer $(19,20)$. Our results also showed that B7-H6 is widely expressed in various hematologic malignancies (data not shown) including B-cell lymphomas, in both primary tumor cells and B-cell lymphoma cell lines. However, it remains to be determined whether B7-H6 expression is associated with outcome in a large cohort of patients with lymphoma.

In this study, we first generated and validated a B-cell lymphoma cell line with B7-H6 knockdown using lentivirus transduction of CA46. B7-H6 expression was significantly decreased at the mRNA and protein level in CA46 cells. The B7-H6 knockdown was specific and efficient, and the B-cell lymphoma cell model was used in the subsequent studies.

Uncontrolled proliferation is one of the important hallmarks of tumor cells (25). Previous studies found that B7-H6 expression is positively correlated with ovarian cancer progression (4), but to date no similar study has been published in B-cell lymphoma. 
We found that B7-H6 silencing inhibited the proliferation and colony formation of lymphoma cells and the expression of Survivin, PCNA and c-Myc was decreased in CA46shB7-H6 cells. Survivin and PCNA are important molecular markers for proliferation and expressed in $\mathrm{G} 2 / \mathrm{M}$ and $\mathrm{S}$ phase, respectively $(26,27)$. Translocation involving c-Myc is characteristic of Burkitt lymphomas (28). The inhibition of cell proliferation might be through downregulation of PCNA, Survivin and c-Myc, however, the molecular mechanisms need further investigations.

Resistance to cell death is also a characteristic of tumor cells (25). Previous studies suggested that high soluble B7-H6 was associated with chemoresistance in neuroblastoma (6). To determine whether B7-H6 silencing influences apoptosis in CA46 cells, we measured the apoptotic rates induced by serum starving and chemotherapeutic drugs. We measured an increased rate of apoptosis in the B7-H6 knockdown group. $\mathrm{Bcl}-2 / \mathrm{Bcl}-\mathrm{xL}$ and $\mathrm{Bax} / \mathrm{Bad}$ are important members of Bcl-2 family, which plays a role in cell apoptosis $(29,30)$. The main function of $\mathrm{Bcl}-2 / \mathrm{Bcl}-\mathrm{xL}$ is to prevent the release of proapoptotic molecules leaking into cytosol to block apoptosis, and the role of $\mathrm{Bax} / \mathrm{Bad}$ is quite the opposite (30). The induction of apoptosis is influenced by the ratio of pro-apoptotic to anti-apoptotic molecules. Our data showed that the expression of Bcl-2 and Bcl-xL were decreased, and the expression of Bax and Bad was increased in CA46shB7-H6 group. Moreover, the expression of Caspase- 8 and -3 , which act as initiator and executor of apoptosis, respectively, was decreased after B7-H6 silencing (31). Thus, our study indicates that knockdown of B7-H6 induces apoptosis and chemotherapeutic susceptibility in lymphoma cells through downregulation of anti-apoptotic proteins, upregulation of pro-apoptotic proteins and activation of Caspase- 8 and -3 .

Disturbance of cell cycle regulation has been shown to be responsible for the initiation and development of solid and hematologic tumors (32-34). We assessed cell cycle distribution and found that B7-H6 silencing induced cell cycle arrest in G0/G1 phase. The Cyclin D1-CDK4/6-Rb pathway is a key regulator of the $\mathrm{G} 1 / \mathrm{S}$ transition $(35,36)$. Cyclin D1 interacts with CDK4/6 to form a complex, which phosphorylates and inactivates $\mathrm{Rb}$ (34). The phosphorylated $\mathrm{Rb}$ protein is released from the transcription factor E2F to promote G1/S transition (34). We found that knockdown of B7-H6 led to decreased expressions of Cyclin D1, CDK4, CDK6, pRb proteins and to increased expression of $\mathrm{Rb}$. The $\mathrm{p} 21$ protein is a CDK inhibitor that binds to the Cyclin D1-CDK4/6 complex and mediates cell G1 cycle arrest (37). As shown in Fig. 5C, the expression of p21 was reduced after B7-H6 silencing. These data suggest that the cell cycle might be regulated by B7-H6 through the Cyclin D1-CDK4/6-Rb axis and p21.

Invasion and metastasis are important hallmarks of tumors (25). Several studies reported that B7-H6 is associated with metastasis in ovarian cancer (4) and neuroblastoma (6). In our study, we observed that B7-H6 silencing inhibited the migratory and invasive properties of CA46 cells. Since MMP-2 and MMP-9 are correlated with invasion and metastasis in lymphomas and solid tumors $(38,39)$, we measured the expressions of these proteins and found them to be decreased in CA46shB7-H6 cells. This observation indicates that inhibition of cell migration and invasion after B7-H6 silencing might be related to downregulation of MMP-2 and MMP-9.
STAT3 plays a critical role in cancer progression (40) and is a promising target for the treatment of multiple malignancies including lymphoma $(22,23,40)$. Inhibition of STAT3 results in decreased proliferation and increased apoptosis in cancer cells (40). In this study, B7-H6 silencing inhibited the activation of STAT3 and ERK1/2. We reactivated the STAT3 pathway with IL-6 and measured downstream targets of STAT3, including Cyclin D1, Bcl-xL and ERK1/2 (40). The data indicated that knockdown of B7-H6 led to dephosphorylation of STAT3 and downregulation of its target proteins. Thus, our data support a model in which B7-H6 inhibition is responsible for decreased tumor cell oncogenicity and increased apoptosis via abrogation of the STAT3 pathway.

Above all, this study confirmed that B7-H6 was widely expressed in lymphomas. Knockdown of B7-H6 not only suppressed cell proliferation, migration and invasion ability, but also enhanced G0/G1 cycle arrest, apoptosis and chemosensitivity in lymphoma cells. This study indicated that B7-H6 inhibition exerts antitumor effects via the inactivation of the STAT3 pathway. These findings suggest that B7-H6 may serve as a therapeutic target in lymphomas.

\section{References}

1. Forman D, Bray F and Brewster D: Cancer Incidence in Five Continents Vol. X (eletronic version). IARC, Lyon, 2013. http:// ci5.iarc.fr/Default .aspx. Accessed January 2, 2014.

2. Zappasodi R, de Braud F and Di Nicola M: Lymphoma immunotherapy: Current status. Front Immunol 6: 448, 2015.

3. Brandt CS, Baratin M, Yi EC, Kennedy J, Gao Z, Fox B Haldeman B, Ostrander CD, Kaifu T, Chabannon C, et al: The B7 family member B7-H6 is a tumor cell ligand for the activating natural killer cell receptor NKp30 in humans. J Exp Med 206: 1495-1503, 2009

4. Zhou Y, Xu Y, Chen L, Xu B, Wu C and Jiang J: B7-H6 expression correlates with cancer progression and patient's survival in human ovarian cancer. Int J Clin Exp Pathol 8: 9428-9433, 2015.

5. Wu MR, Zhang T, DeMars LR and Sentman CL: B7H6-specific chimeric antigen receptors lead to tumor elimination and host antitumor immunity. Gene Ther 22: 675-684, 2015.

6. Semeraro M, Rusakiewicz S, Minard-Colin V, Delahaye NF, Enot D, Vély F, Marabelle A, Papoular B, Piperoglou C, Ponzoni M, et al: Clinical impact of the NKp30/B7-H6 axis in high-risk neuroblastoma patients. Sci Transl Med 7: 283ra55, 2015.

7. Kellner C, Maurer T, Hallack D, Repp R, van de Winkel JG, Parren PW, Valerius T, Humpe A, Gramatzki M and Peipp M: Mimicking an induced self phenotype by coating lymphomas with the NKp30 ligand B7-H6 promotes NK cell cytotoxicity. J Immunol 189: 5037-5046, 2012.

8. Wu MR, Zhang T, Gacerez AT, Coupet TA, DeMars LR and Sentman CL: B7H6-specific bispecific T cell engagers lead to tumor elimination and host antitumor immunity. J Immunol 194: 5305-5311, 2015.

9. Mir MA and Agrewala JN: Signaling through CD80: An approach for treating lymphomas. Expert Opin Ther Targets 12: 969-979, 2008.

10. Philips GK and Atkins M: Therapeutic uses of anti-PD-1 and anti-PD-L1 antibodies. Int Immunol 27: 39-46, 2015.

11. Leung J and Suh WK: The CD28-B7 family in anti-tumor immunity: Emerging concepts in cancer immunotherapy. Immune Netw 14: 265-276, 2014.

12. Seliger B and Quandt D: The expression, function, and clinical relevance of B7 family members in cancer. Cancer Immunol Immunother 61: 1327-1341, 2012.

13. Zhang W, Wang Y, Wang J, Dong F, Zhu M, Wan W, Li H, Wu F, Yan $\mathrm{X}$ and $\mathrm{Ke} \mathrm{X}$ : B7-H3 silencing inhibits tumor progression of mantle cell lymphoma and enhances chemosensitivity. Int J Oncol 46: 2562-2572, 2015.

14. Li Y, Wang J, Li C and Ke XY: Contribution of PD-L1 to oncogenesis of lymphoma and its RNAi-based targeting therapy. Leuk Lymphoma 53: 2015-2023, 2012. 
15. Kaifu T, Escalière B, Gastinel LN, Vivier E and Baratin M: B7-H6/NKp30 interaction: A mechanism of alerting NK cells against tumors. Cell Mol Life Sci 68: 3531-3539, 2011.

16. Zhang T, Wu MR and Sentman CL: An NKp30-based chimeric antigen receptor promotes $T$ cell effector functions and antitumor efficacy in vivo. J Immunol 189: 2290-2299, 2012.

17. Schlecker E, Fiegler N, Arnold A, Altevogt P, Rose-John S, Moldenhauer G, Sucker A, Paschen A, von Strandmann EP, Textor S, et al: Metalloprotease-mediated tumor cell shedding of B7-H6, the ligand of the natural killer cell-activating receptor NKp30. Cancer Res 74: 3429-3440, 2014.

18. Fiegler N, Textor S, Arnold A, Rölle A, Oehme I, Breuhahn K, Moldenhauer $G$, Witzens-Harig $M$ and Cerwenka $A$ : Downregulation of the activating NKp30 ligand B7-H6 by HDAC inhibitors impairs tumor cell recognition by NK cells. Blood 122 : 684-693, 2013.

19. Chen XJ, Shen J, Zhang GB and Chen WC: B7-H6 protein expression has no prognostic significance in human gastric carcinoma. Pathol Oncol Res 20: 203-207, 2014

20. Zhang X, Zhang G, Qin Y, Bai R and Huang J: B7-H6 expression in non-small cell lung cancers. Int J Clin Exp Pathol 7: 6936-6942, 2014.

21. Baran-Marszak F, Boukhiar M, Harel S, Laguillier C, Roger C, Gressin R, Martin A, Fagard R, Varin-Blank N, AjchenbaumCymbalista F, et al: Constitutive and B-cell receptor-induced activation of STAT3 are important signaling pathways targeted by bortezomib in leukemic mantle cell lymphoma. Haematologica 95: 1865-1872, 2010

22. Munoz J, Dhillon N, Janku F, Watowich SS and Hong DS: STAT3 inhibitors: Finding a home in lymphoma and leukemia. Oncologist 19: 536-544, 2014.

23. Soldini D, Montagna C, Schüffler P, Martin V, Georgis A, Thiesler T, Curioni-Fontecedro A, Went P, Bosshard G, Dehler S, et al: A new diagnostic algorithm for Burkitt and diffuse large B-cell lymphomas based on the expression of CSE1L and STAT3 and on MYC rearrangement predicts outcome. Ann Oncol 24: 193-201, 2013.

24. Lu S, Gao Y, Huang X and Wang X: GYY4137, a hydrogen sulfide $\left(\mathrm{H}_{2} \mathrm{~S}\right)$ donor, shows potent anti-hepatocellular carcinoma activity through blocking the STAT3 pathway. Int J Oncol 44: 1259-1267, 2014.

25. Hanahan D and Weinberg RA: Hallmarks of cancer: The next generation. Cell 144: 646-674, 2011.

26. He C, Liu Z, Ji J and Zhu H: Prognostic value of survivin in patients with non-Hodgkin's lymphoma: A meta-analysis. Int J Clin Exp Med 8: 5847-5854, 2015.
27. Wang SC: PCNA: A silent housekeeper or a potential therapeutic target? Trends Pharmacol Sci 35: 178-186, 2014.

28. Said J, Lones M and Yea S: Burkitt lymphoma and MYC: What else is new? Adv Anat Pathol 21: 160-165, 2014.

29. Brinkmann $\mathrm{K}$ and Kashkar H: Targeting the mitochondrial apoptotic pathway: A preferred approach in hematologic malignancies? Cell Death Dis 5: e1098, 2014.

30. Hardwick JM and Soane L: Multiple functions of BCL-2 family proteins. Cold Spring Harb Perspect Biol 5: a8722, 2013.

31. Shalini S, Dorstyn L, Dawar S and Kumar S: Old, new and emerging functions of caspases. Cell Death Differ 22: 526-539, 2015.

32. Yang WJ, Yu Z and Qiu LG: Research advances of signal pathway in the pathogenesis of mantle cell lymphoma. Zhonghua Xue Ye Xue Za Zhi 34: 1073-1075, 2013 (In Chinese).

33. Bonn BR, Krieger D and Burkhardt B: Cell cycle regulatory molecular profiles of pediatric T-cell lymphoblastic leukemia and lymphoma. Leuk Lymphoma 53: 557-568, 2012.

34. Tamura K: Development of cell-cycle checkpoint therapy for solid tumors. Jpn J Clin Oncol 45: 1097-1102, 2015.

35. Besson A, Dowdy SF and Roberts JM: CDK inhibitors: Cell cycle regulators and beyond. Dev Cell 14: 159-169, 2008.

36. Migliaccio I, Di Leo A and Malorni L: Cyclin-dependent kinase 4/6 inhibitors in breast cancer therapy. Curr Opin Oncol 26: 568-575, 2014.

37. Dutto I, Tillhon M, Cazzalini O, Stivala LA and Prosperi E: Biology of the cell cycle inhibitor p21(CDKN1A): Molecular mechanisms and relevance in chemical toxicology. Arch Toxicol 89: 155-178, 2015

38. Hazar B, Polat G, Seyrek E, Bağdatoğlğlu O, Kanik A and Tiftik N: Prognostic value of matrix metalloproteinases (MMP-2 and MMP-9) in Hodgkin's and non-Hodgkin's lymphoma. Int J Clin Pract 58: 139-143, 2004

39. Suminoe A, Matsuzaki A, Hattori H, Koga Y, Ishii E and Hara T: Expression of matrix metalloproteinase (MMP) and tissue inhibitor of MMP (TIMP) genes in blasts of infant acute lymphoblastic leukemia with organ involvement. Leuk Res 31: 1437-1440, 2007

40. Xiong A, Yang Z, Shen Y, Zhou J and Shen Q: Transcription factor STAT3 as a novel molecular target for cancer prevention. Cancers (Basel) 6: 926-957, 2014. 